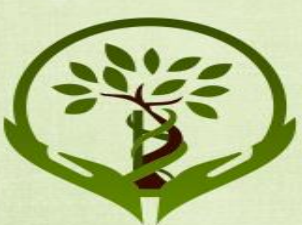

ISSN: $2179-6572$

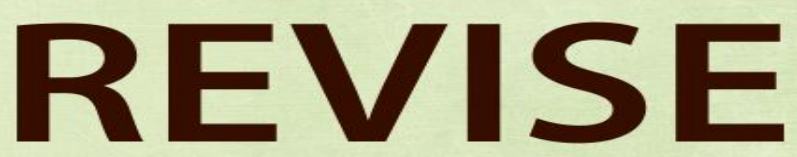

Revista integrativa em inovação tecnológica nas ciências da saúde

\title{
Artigo: PRODUÇÃO DE ALIMENTO FUNCIONAL A BASE DE AMÊNDOAS DE CUPUAÇU
}
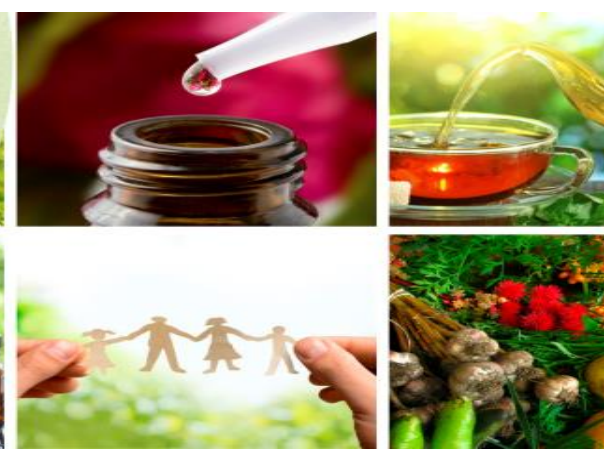

Helyene Silva Santos

Valdineide Souza da Silva

Valdirene Lima Pinheiro

Ferlando Lima Santos

UFRB

\section{RESUMO}

A busca por um alimento de melhor qualidade tem sido uma preocupação de grande parte da população que na procura pela manutenção da saúde tem procurado consumir produtos que ofereçam melhores propriedades nutricionais. Nesta expectativa, a indústria tem dado ênfase a alimentos funcionais, os quais além de dispor dos ingredientes nutricionais básicos desempenham alguma função metabólica ou efeitos benéficos para a saúde. O objetivo deste estudo foi desenvolver o bombom de cupuaçu (cupulate) com recheio de banana e amendoim, tendo como principais atributos as fibras, mais precisamente a fibra solúvel pectina, e os ácidos graxos insaturados. $\mathrm{O}$ bombom foi elaborado no Laboratório de Tecnologia de Alimentos da Universidade Federal do Recôncavo da Bahia (UFRB) e a análise sensorial deste foi feita com 60 adultos de ambos os sexos em uma Feira de Alimentos Funcionais, realizada no campus da universidade. Os resultados obtidos revelaram que $98 \%$ das avaliações estavam na área de aceitação, indicando que o produto desenvolvido possui alta capacidade de comercialização, sendo uma alternativa mais saudável no ramo da alimentação.

Palavras-chave: bombom, cupulate, funcional, cupuaçu, fibras.

\begin{abstract}
The search for a better-quality food has been a concern of a large part of the population that in the search for health maintenance has sought to consume products that offer better nutritional properties. In this expectation, the industry has emphasized functional foods, which in addition to having the basic nutritional ingredients play some metabolic function or beneficial health effects. The aim of this study was to develop cupulate with banana and peanut filling, having as main attributes fibers, more precisely soluble pectin fiber, and unsaturated fatty acids. The candy was prepared at the Food Technology Laboratory of the Federal University of Recôncavo da Bahia (UFRB) and its sensory analysis was performed with 60 adults of both sexes at a Functional Food Fair, held on the university campus. The results showed that $98 \%$ of the evaluations were in the acceptance area, indicating that the developed product has high commercialization capacity, being a healthier alternative in the food industry.

Keywords: bonbon, cupulate, functional, cupuaçu, fibers.
\end{abstract}

Alimentos funcionais. Revista Revise, vol. 3, no fluxo contínuo, p.173-186. 

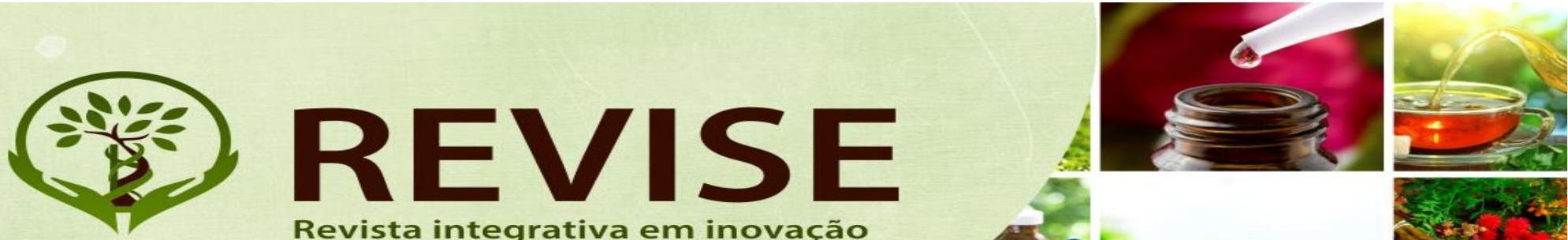

Revista integrativa em inovação tecnológica nas ciências da saúde

\section{INTRODUÇÃO}

Atualmente grande parte da população tem demonstrado preocupação com a saúde, assim, a busca por uma alimentação melhor tem ganhado um papel importante na vida das pessoas. Sendo assim houve uma procura por alimentos funcionais, estes alimentos consistem em: aqueles semelhantes em aparência ao alimento convencional, consumido como parte de uma alimentação normal e possui a capacidade de produzir efeitos metabólicos ou fisiológicos desejáveis na manutenção da saúde. ( SGARBIERI e PACHECO). Entre os fatores-chave que explicam o êxito dos alimentos funcionais, Hasler (2000) ratifica ser a preocupação crescente pela saúde e pelo bem estar, mudanças na regulamentação dos alimentos e a crescente comprovação científica das relações existentes entre dieta e saúde.

Os alimentos funcionais vêm adquirindo uma presença crescente na indústria de alimentos, tendo faturado 2,5 bilhões de dólares, em 2003 (SBAF, 2007). Eles são a nova tendência do poderoso mercado alimentício neste início do século XXI (HEASMAN e MELLENTIN, 2001) Nesta perspectiva, houve a iniciativa de utilizar as amêndoas do cupuaçu como matéria prima base para a elaboração deste produto devido a constatação de que o cupulate - produto análogo ao chocolate desenvolvido por volta do ano de 1990 - figure como um produto semelhante ao chocolate quanto ao sabor, cor e textura, entretanto com algumas propriedades nutricionais distintas deste, o que confere novidade ao produto. Segundo Severiano (2011), as sementes, matéria-prima do cupulate, são ricas em lipídios, proteínas e calorias e depois de fermentadas, torradas e moídas geram um produto, que garantem os especialistas, não deixa nada a desejar ao chocolate tradicional, com composição química e nutricional bem semelhante. A 

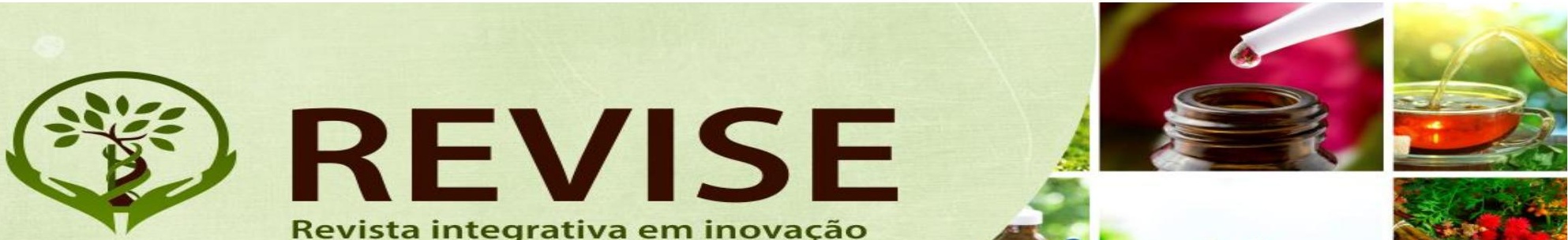

Revista integrativa em inovação tecnológica nas ciências da saúde

ISSN: $2179-6572$

diferença fica por conta do teor de manteiga que é ligeiramente superior ao do produto de cacau.

O cupuaçu possui sementes com $1 / 3$ de teor de polifenólicos e taninos que as sementes do cacau (PUGLIESE, 2010). Aliado ao cupuaçu, as propriedades funcionais do produto serão enriquecidas com a banana e o amendoim, tendo em vista que são, respectivamente, uma fruta e uma leguminosa produtora de grãos com grande abundância no Recôncavo Baiano e de fácil acesso para a população. Segundo os dados da Revista Brasileira de Produtos Agroindustriais, por ser rica em fibras, a banana ajuda a dar saciedade e melhorar funcionamento intestinal.

A banana é composta basicamente de água, proteína, carboidrato, além de um regular teor de cálcio, ferro, cobre, zinco, iodo, manganês e cobalto. (LIMA, NEBRA e QUEIROZ, 2000) O amendoim por sua vez é uma rica fonte de ácidos gráxicos monoinsaturados, conhecidos como gordura do bem, que ajudam a perder peso, pois são responsáveis por manter o nível de açúcar no sangue estável e ativar o metabolismo da queima de gorduras, além de ajudar a converter os estoques de gordura corporal em energia. É uma leguminosa de alto valor nutritivo com um perfil vitamínico invejável e uma grande quantidade de proteínas vegetais sendo de 25 a $30 \%$ da sua composição (MARCHIORE). Este trabalho teve como objetivo a elaboração de bombom funcional a partir das amêndoas de cupuaçu com recheio de banana e amendoim.

\section{Material e Métodos}

O bombom foi desenvolvido no laboratório de Tecnologia de Alimentos da Universidade Federal do Recôncavo da Bahia, Centro de Ciências da Saúde, na cidade de Santo Antônio de Jesus-Ba. Na elaboração do produto foram utilizados os ingredientes apresentados conforme a Tabela 1. 

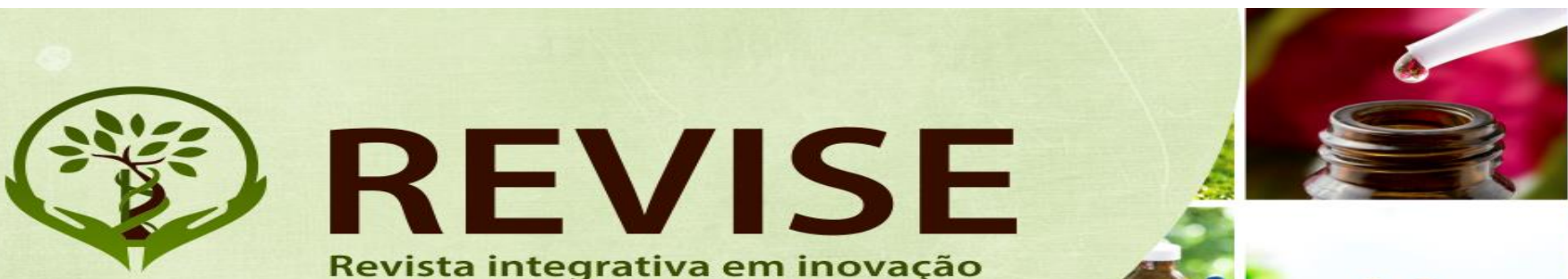

Revista integrativa em inovação tecnológica nas ciências da saúde

Tabela 1 - Ingredientes utilizados na formulação do bombom

INGREDIENTES

QUANTIDADE

\begin{tabular}{|c|c|}
\hline Leite em pó integral & $35 \mathrm{~g}$ \\
\hline Sementes de cupuaçu trituradas & $35 \mathrm{~g}$ \\
\hline Água & $100 \mathrm{~g}$ \\
\hline Açúcar & $40 \mathrm{~g}$ \\
\hline Gordura vegetal & $3 \mathrm{~g}$ \\
\hline Amendoim & $20 \mathrm{~g}$ \\
\hline Banana & $30 \mathrm{~g}$ \\
\hline
\end{tabular}

Os utensílios empregados no preparo foram: colheres, liquidificador, balança analítica, panela, peneira, espátula de silicone e fogão.

\section{Ingredientes Quantidade}

Leite em pó integral 35g Sementes de cupuaçu trituradas 35g Água 100g Açúcar 40g Gordura vegetal 3g Amendoim 20g Banana 30g. Para elaboração do bombom foi

Alimentos funcionais. Revista Revise, vol. 3, no fluxo contínuo, p.173-186. 

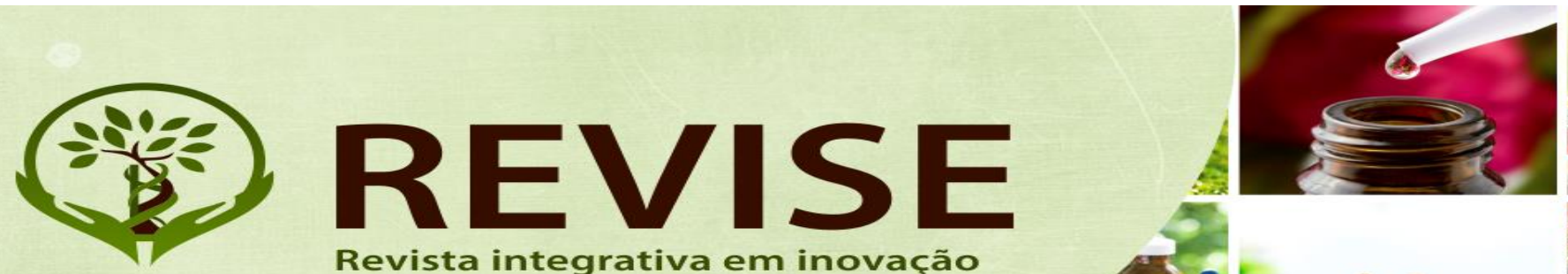

Revista integrativa em inovação tecnológica nas ciências da saúde

ISSN: 2179-6572

utilizado sementes do cupuaçu pré-torradas e em seguida estas foram levadas ao forno alto com temperatura de $180^{\circ}$, por 10 minutos. Passando esse período pesou as sementes e obteve 43,12g. Em seguida elas foram trituradas no liquidificador, depois peneiradas e novamente pesadas obteve-se $35 \mathrm{~g}$, pois alguns resíduos foram retirados.

Obtido essa farinha das amêndoas, adicionou $35 \mathrm{~g}$ de leite em pó integral, $40 \mathrm{~g}$ de açúcar, $100 \mathrm{ml}$ de água e $3 \mathrm{~g}$ de gordura vegetal e levou ao fogo baixo. Essa mistura ficou por aproximadamente 10 minutos no fogo, até soltar do fundo da panela. Depois de pronto foi colocado num recipiente para depois modelar os bombons. Para o recheio, levou a banana ao fogo, com açúcar até dar o ponto de um doce de banana, em seguida adicionou o amendoim, torrado e triturado formando uma mistura homogênea e consistente para rechear o bombom. 

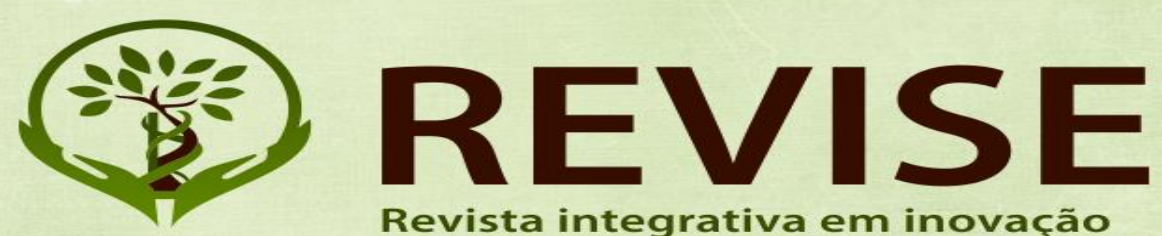

Revista integrativa em inovação tecnológica nas ciências da saúde ISSN: $2179-6572$

Fluxograma: Desenvolvimento do produto
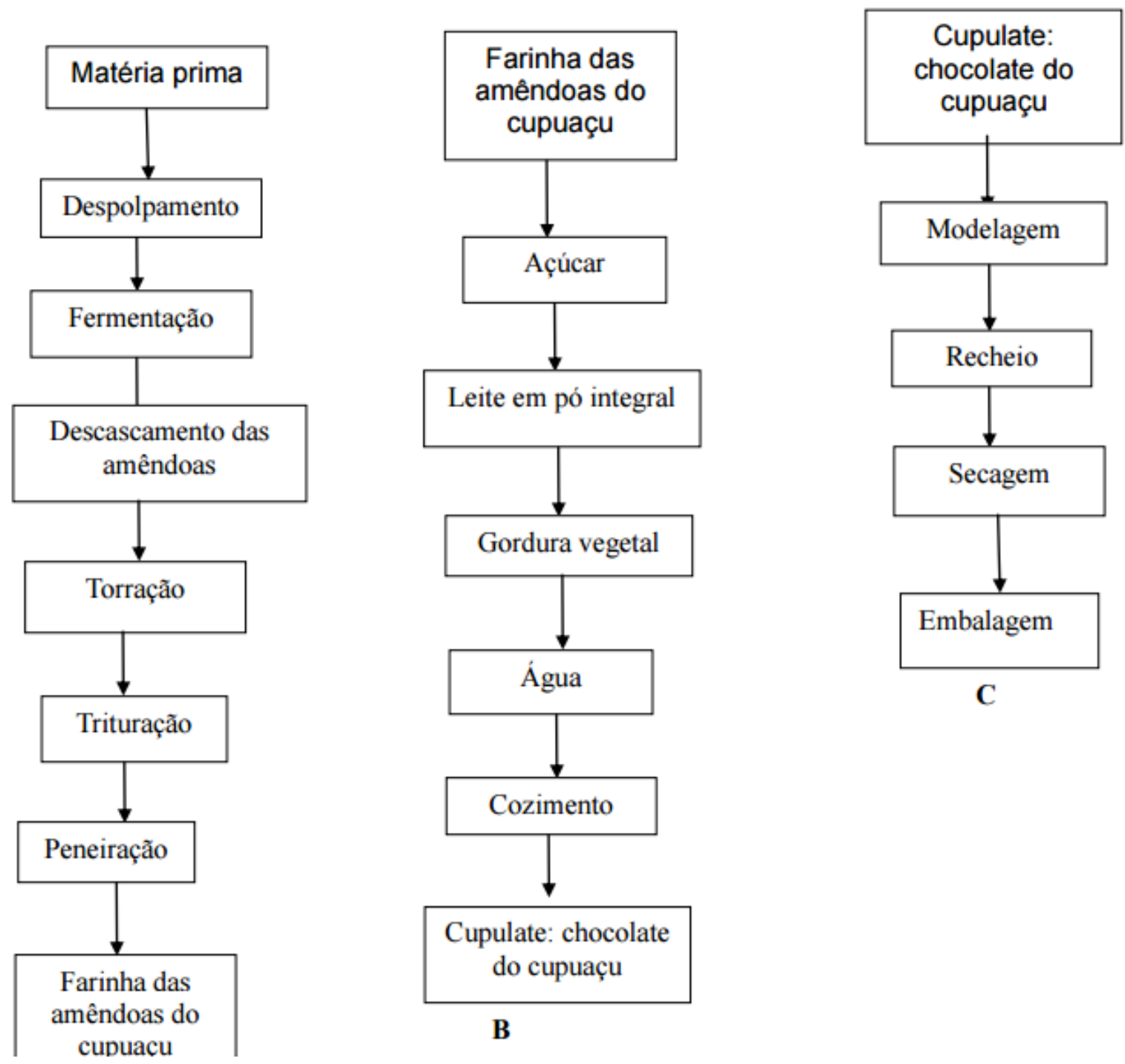

C

\section{B}



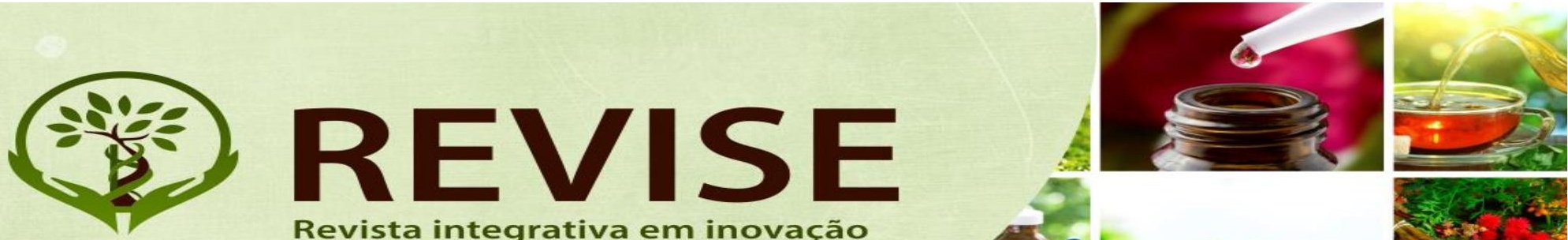

Revista integrativa em inovação tecnológica nas ciências da saúde

\section{FLUXOGRAMA}

Desenvolvimento do produto A

O fluxograma acima apresenta o desenvolvimento do produto, sendo que o fluxograma A, refere-se ao processo de obtenção da farinha do cupuaçu. A imagem B demonstra as etapas do processo para a elaboração do chocolate de cupuaçu (cupulate), já o fluxograma $\mathrm{C}$, refere-se à fabricação do produto, o bombom de cupulate. Inicialmente foi realizado um pré-teste, nesse momento os primeiros produtos elaborados foram oferecidos para algumas pessoas a fim de obter as primeiras impressões sobre o produto, a partir desse pré-teste foi possível fazer algumas alterações para melhorar a Matéria prima Despolpamento Fermentação Descascamento das amêndoas Torração Trituração Peneiração Farinha das amêndoas do cupuaçu Farinha das amêndoas do cupuaçu Açúcar Leite em pó integral Gordura vegetal Água Cozimento Cupulate: chocolate do cupuaçu Cupulate: chocolate do cupuaçu Modelagem Recheio Secagem Embalagem B C qualidade do produto.

A avaliação sensorial foi realizada por meio do método de análise sensorial afetivo, cujo objetivo foi avaliar a aceitação dos consumidores em relação a preparação. Nesse sentido, foi feito um teste de aceitação no qual os interessados responderam a um questionário de avaliação de perfil contendo informações tais como faixa etária e sexo, detectando-se aspectos relevantes como frequência de consumo de bombom e utilizando como parâmetros a escala hedônica estruturada de nove pontos, que variou entre "gostei extremamente" (nota 9) a "desgostei extremamente" nota (1), e a escala de atitude que variou entre "comeria isto sempre que tivesse oportunidade" nota (9) a "comeria isso se fosse forçado" (nota 1). Além de escalas de 1 a 9 para avaliar sabor, cor e comparação com outros produtos que são vendidos no comércio. Posteriormente, os dados coletados foram tabulados e analisados utilizando o programa Excel. Para a interpretação dos 

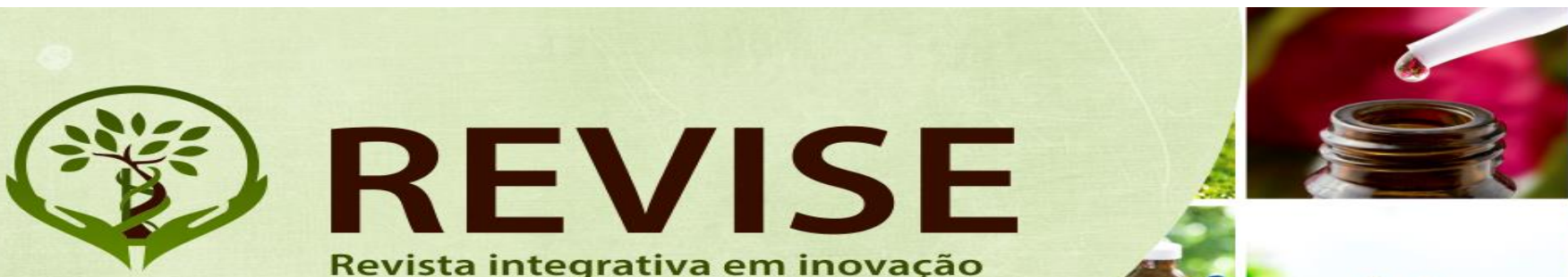

Revista integrativa em inovação tecnológica nas ciências da saúde

ISSN: $2179-6572$

dados, considerou-se que a zona de aceitação é composta pela soma das avaliações que estavam entre variáveis 6 e 9 da escala hedônica de 9 pontos, a indiferença na variável 5 e a zona de rejeição entre as variáveis 1 e 4 .

\section{Resultados e Discussão}

Foi realizado uma análise indireta do produto, por meio de tabelas de composições químicas e por meio de artigos científicos. O produto apresentou os valores demonstrados na Tabela 2

\begin{tabular}{|c|c|}
\hline \multicolumn{2}{|l|}{ Tabela 2 - tabela de composição centesimal da formulação do bombom. } \\
\hline Nutriente Porção (25g) \\
\hline Valor energético & $193,92 \mathrm{Kcal}$ \\
\hline Carboidratos & $10,39 \mathrm{~g}$ \\
\hline Proteinas & $2,62 \mathrm{~g}$ \\
\hline Lipideos & $4,9 \mathrm{~g}$ \\
\hline Fibras & $1,34 \mathrm{~g}$ \\
\hline Gorduras totais & $2,95 \mathrm{~g}$ \\
\hline
\end{tabular}

Os resultados alcançados sugerem um produto bastante nutritivo e que atende ao desejo dos consumidores. Porém é importante destacar que o cupulate, chocolate proveniente das amêndoas do cupuaçu ainda é um alimento considerado novo. Sendo assim não permitiu que houvesse comparação por falta de dados na literatura.

Através da degustação, em que cada um dos provadores recebeu uma amostra correspondente a $25 \mathrm{~g}$ da formulação. O julgamento consistiu em atribuir a impressão geral sobre os principais aspectos, tais como sabor e cor da formulação. Participaram da análise sensorial 60 pessoas, sendo 40 do sexo feminino e 20 do sexo masculino, média de 25,6 anos de idade.

Em relação ao consumo de chocolate a maioria dos participantes $31,7 \%$ afirmaram que consomem chocolate de $2 / 3$ vezes por semana, seguido por $25 \%$ das pessoas que fazer o uso desse produto diariamente. Os que consomem uma vez por 

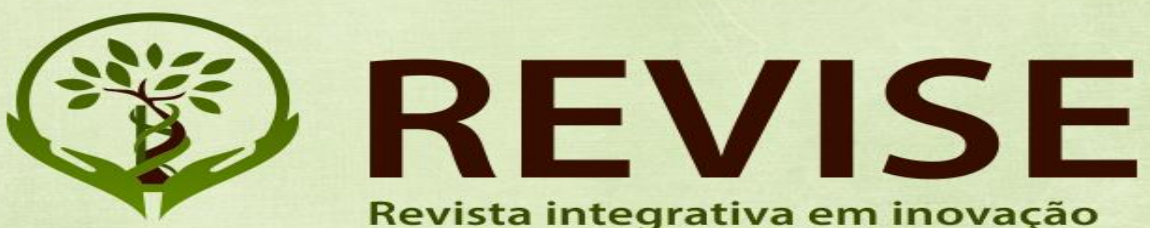

Revista integrativa em inovação tecnológica nas ciências da saúde

ISSN: 2179-6572

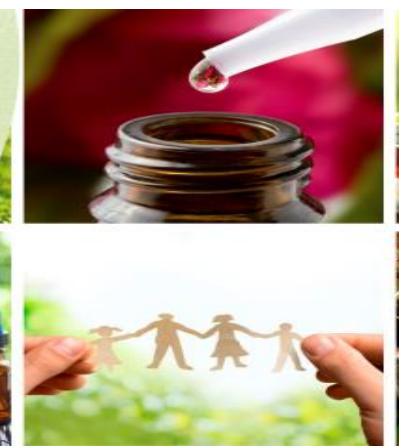

semana foi $23,3 \%$ dos degustadores, quinzenalmente $8,3 \%$ e mensalmente $10 \%$, como mostra o gráfico 1.

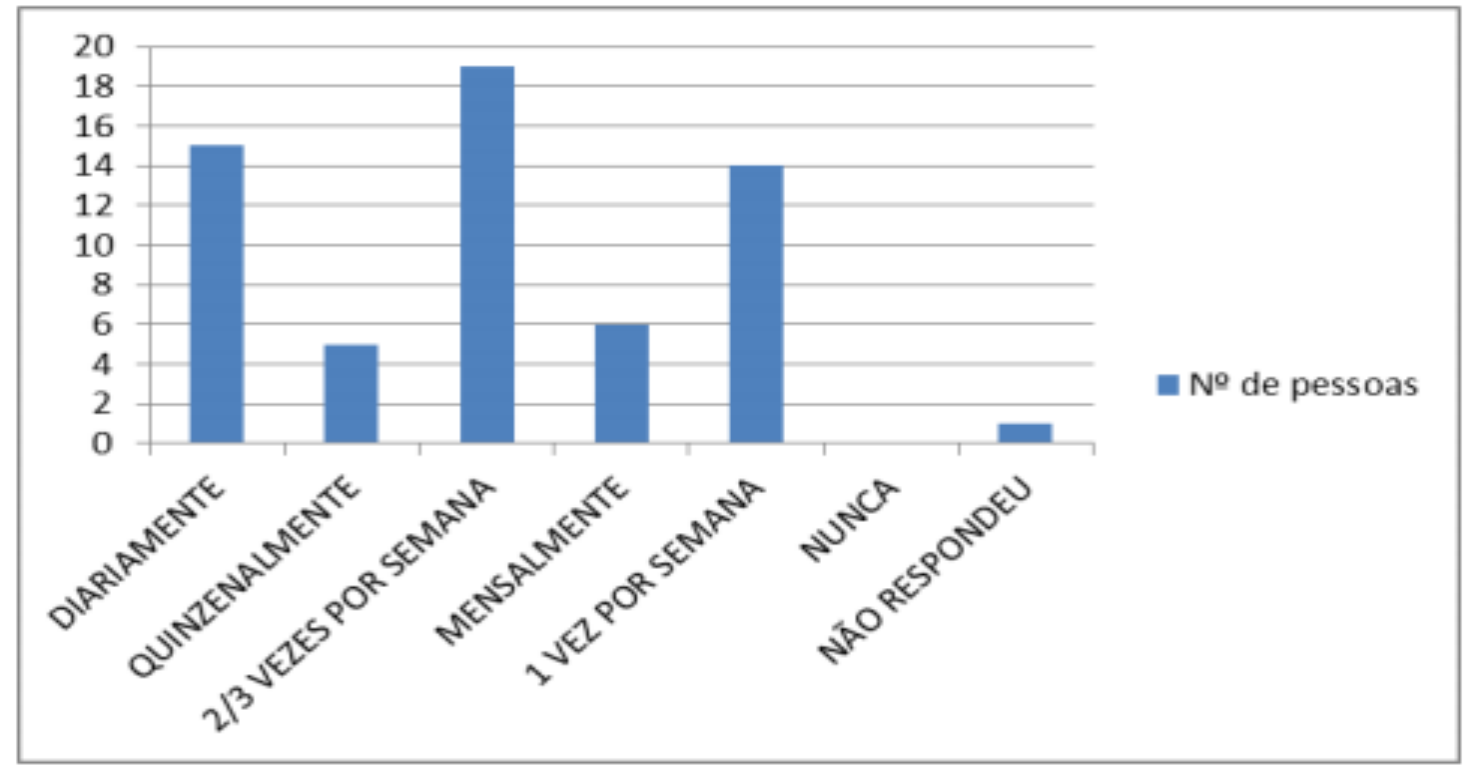

Gráfico 1. Frequência de consumo de chocolate relatada pelos provadores.

Os resultados encontrados apresentam uma boa aceitação do bombom de cupulate, considerando que os provadores possuem o hábito de consumir o chocolate mais de uma vez por semana, aprovaram a proposta do cupulate, o chocolate de cupuaçu. No gráfico 2 pode ser observado que 56,7\% dos participantes gostaram muito do produto; $20 \%$ gostaram extremamente; $18,3 \%$ gostaram moderadamente: $3,3 \%$ gostaram ligeiramente e $1,7 \%$ acharam indiferente.

Alimentos funcionais. Revista Revise, vol. 3, no fluxo contínuo, p.173-186. 

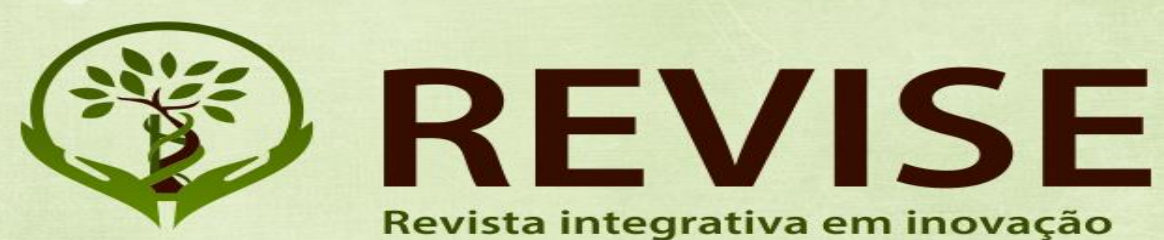

Revista integrativa em inovação tecnológica nas ciências da saúde

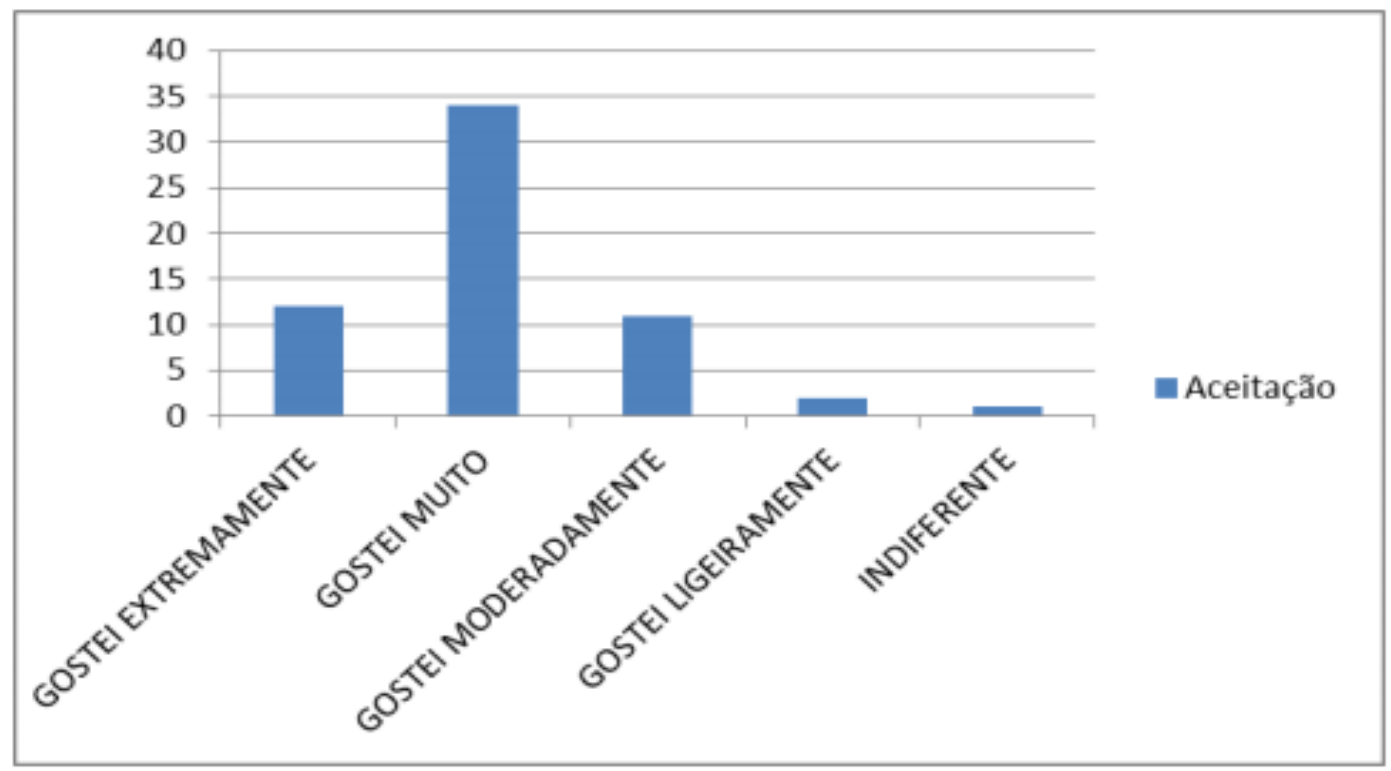

Gráfico 2. Aceitação com relação ao produto.

Analisando o terceiro gráfico, verifica-se que se caso o produto estivesse disponível para ser comercializado $31,7 \%$ dos provadores comeriam sempre que tivesse oportunidade; $25 \%$ comeriam isso frequentemente; comeria o produto muito frequentemente e comeria de vez em quando tiveram um empate sendo $20 \%$ para cada opção; raramente comeria o produto e comeria o produto se tivesse oportunidade tiveram $1,7 \%$ para cada alternativa.

Alimentos funcionais. Revista Revise, vol. 3, no fluxo contínuo, p.173-186. 

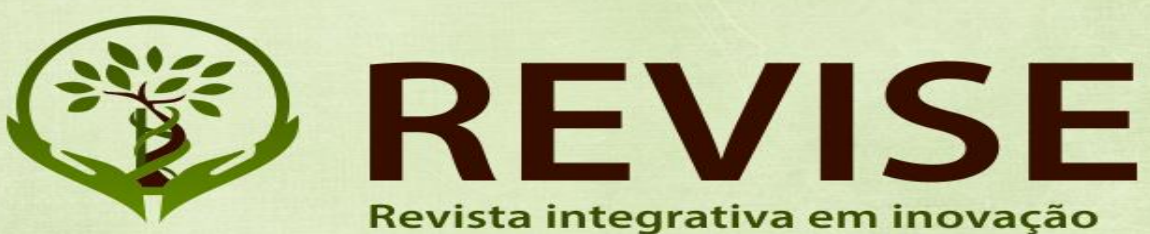

Revista integrativa em inovação tecnológica nas ciências da saúde

ISSN: 2179-6572

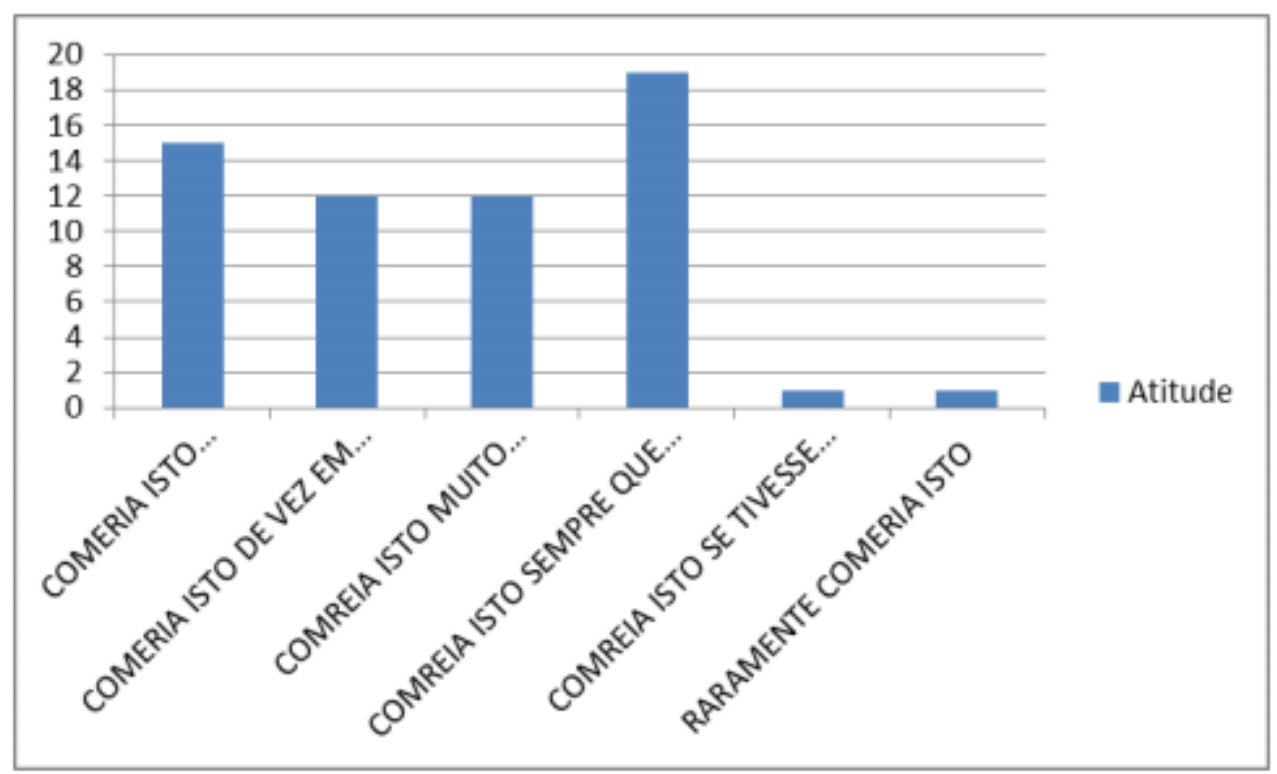

Gráfico 3. Desejo de consumo com relação ao produto.

Em se tratando das características físicas do produto cor e sabor, obteve-se resultados bastantes próximos. Em relação ao sabor $85 \%$ das pessoas que participaram da degustação ficaram na área de 6 a 9, sendo considerado aceito. E para cor 86,7\% ocuparam a zona de 6 a 9. Desta forma considerou-se um produto aceitável. 

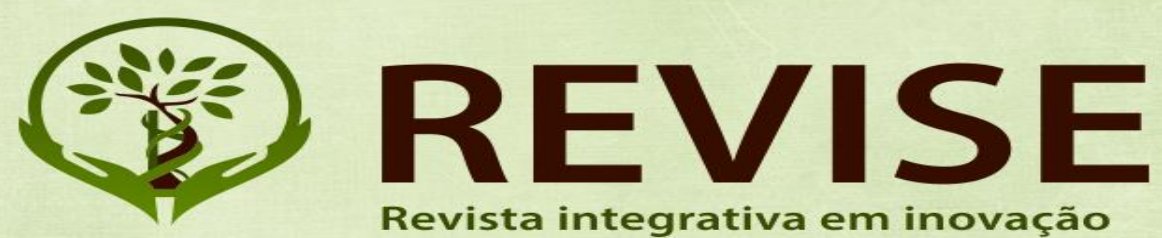

Revista integrativa em inovação tecnológica nas ciências da saúde

ISSN: $2179-6572$

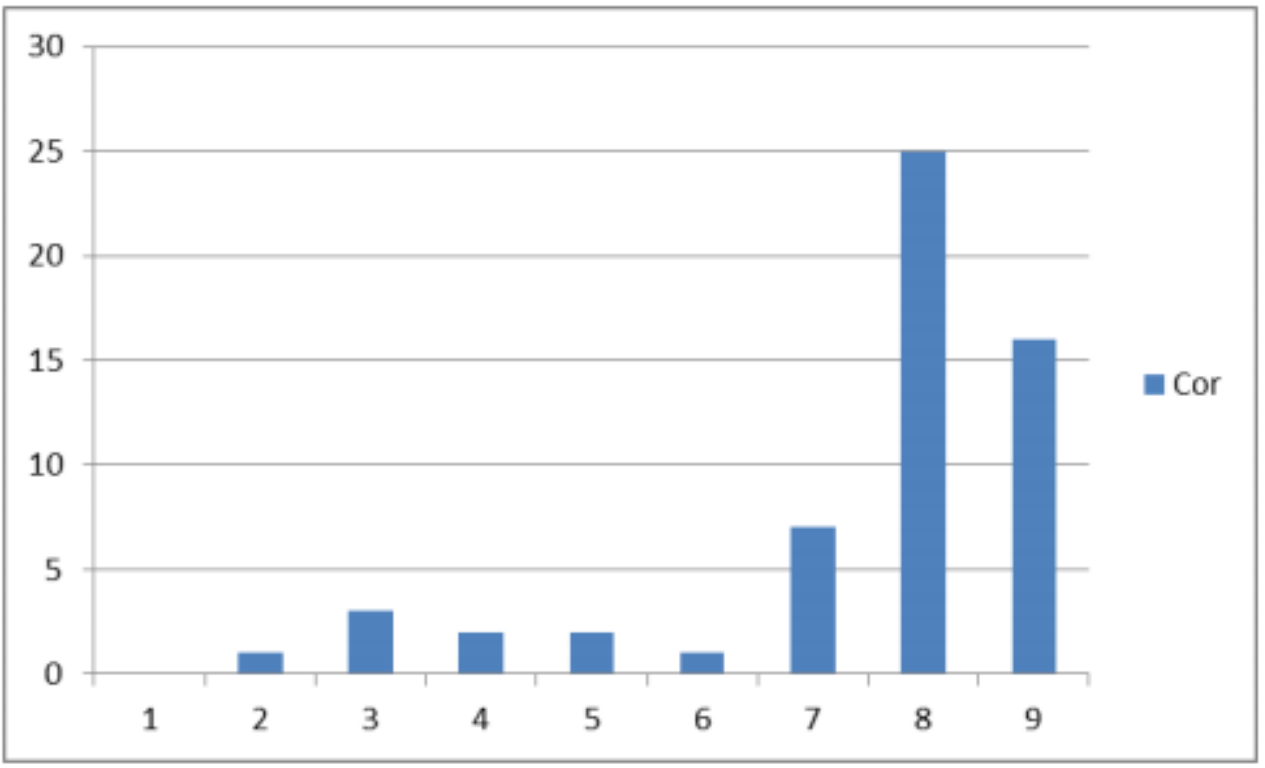

Gráfico 4. Avaliação da cor do produto. 

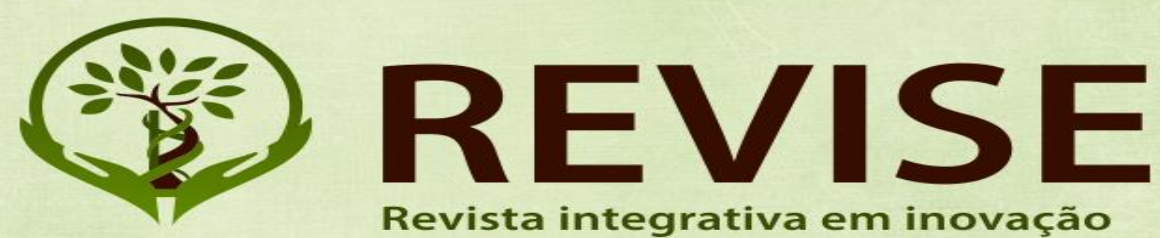

Revista integrativa em inovação tecnológica nas ciências da saúde

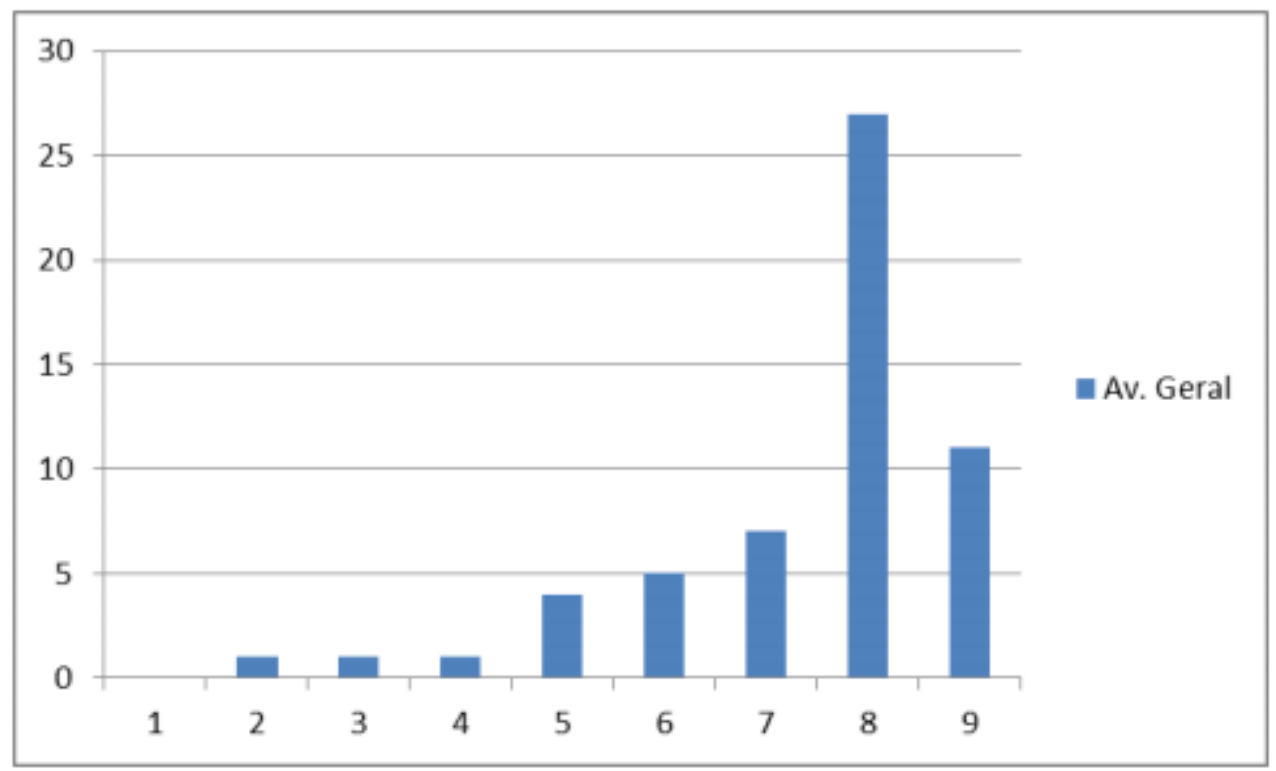

Gráfico 5. Avaliação geral do produto.

Conclusão

A aceitabilidade do bombom de cupulate foi bastante satisfatória, apontando que o produto elaborado apresenta um grande potencial para introdução no mercado. Vale destacar que é necessário mais estudo acerca das propriedades nutricionais das amêndoas do cupuaçu e consequentemente mais estudos sobre a elaboração do cupulate. 

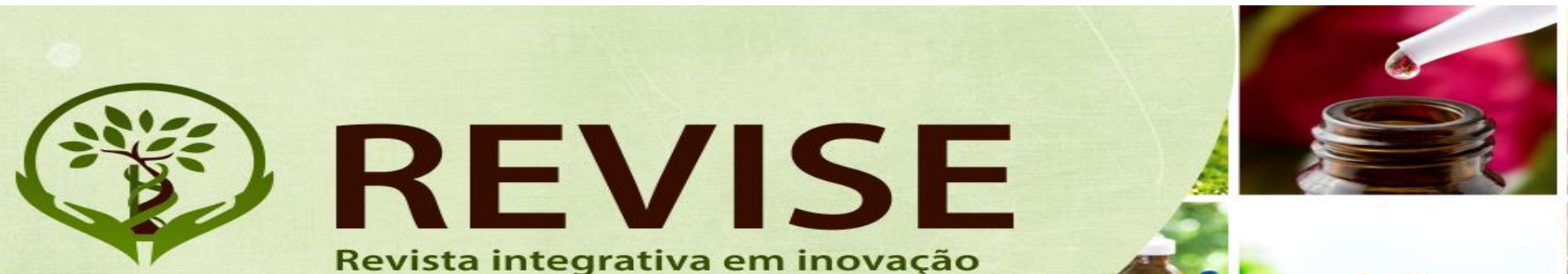

Revista integrativa em inovação tecnológica nas ciências da saúde

\section{Referências}

CARVALHO, Ana Vânia, GARCIA, Nelson Horácio Pezoa, FARFÁN, Jaime Amaya. Proteínas da semente de cupuaçu e alterações devidas à fermentação e à torração. Ciência e Tecnologia de Alimentos. Campinas, 2008.

DURAN, Maria Raquel da Cruz. Os Múltiplos Sentidos do Conhecimento Tradicional: "O caso do cupulate”. Tecnologia e Sociedade - PPGCTS/UFSCar.

GONDIM, Tarcisio Marcos de Souza [etal]. Aspectos da produção de cupuaçu. Rio Branco: Embrapa. 2001.

LIMA, Antônio Barbosa de, NEBRA, Silvia Azucena, QUEIROZ, Marlene Rita de. Aspectos Científico e Tecnológico da Banana. Campina Grande, 2000.

MARCHIORI, Vanderli. Amendoim - Propriedades Funcionais. Associação Brasileira da Indústria de Chocolates, Cacau, Amendoim, Balas e Derivados. São Paulo, 2001.

PUGLIESE. Alexandre Gruber. Compostos fenólicos do Cupuaçu (Theobroma grandiflorum) e do cupulate: Composição e possíveis benefícios. São Paulo, 2010.

RAUD, Cécile. Os Alimentos Funcionais: A Nova Fronteira da Indústria Alimentar, Análise das Estratégias da Danone e da Nestlé no Mercado Brasileiro de Iogurtes. Curitiba, 2008.

SGARBIERI, Valdemiro Carlos, PACHECO, Maria Teresa Bertoldo. Alimentos Funcionais. Campinas - SP: Instituto de Tecnologia de Alimentos.

Alimentos funcionais. Revista Revise, vol. 3, no fluxo contínuo, p.173-186. 